\title{
Dienen Komplementärmedizin und klassische Naturheilkunde dem gesunden Leben oder nur der Heilung von Krankheiten? Besondere Anforderungen an die «evidence-based medicine»
}

\author{
Klaus Michael Meyer-Abich
}

Hamburg, Deutschland

Die herrschende Medizin verdankt ihr Ansehen der Annahme, dass das ärztliche Handeln naturwissenschaftlich begründet sei. Das hiesse: Was der Arzt tut, ist - trotz gelegentlicher Unsicherheiten und Irrtümer - wissenschaftlich richtig und alles Entgegengesetzte wäre wissenschaftlich falsch. Genauere Beobachter hat es deshalb etwa seit den 1960er Jahren zunehmend gewundert, dass beispielsweise bestimmte Operationen oder anderweitige Therapien in verschiedenen Regionen oder Ländern, deren Bevölkerung sich gesundheitlich, wirtschaftlich und in ihren Lebensweisen nicht sonderlich unterscheidet, zwei- oder dreimal so oft stattfinden wie in andern. Dieselben Unterschiede ergeben sich, wenn Gutachtergruppen von Ärzten aus verschiedenen Ländern eine Anzahl von Fällen mit bestimmten Diagnosen unabhängig von den Kosten daraufhin beurteilen, welche Therapie sie für die richtige halten.

Unter der Devise «evidence-based medicine» (EBM) - beweisgestützte Medizin - hat mittlerweile in der herrschenden Medizin selbst eine kritische Untersuchung der Wissenschaftlichkeit des ärztlichen Handelns eingesetzt. Dabei hat sich bestätigt, dass dieses «zu dem naturwissenschaftlichen oder irgendeinem sonstigen Wissen nur einen viel lockereren Bezug hat, als es die offiziellen Verlautbarungen nahe legen wollen» [«...much more loosely connected with scientific or any other form of knowledge than the official rhetoric would suggest», aus 1]. Unter den Medizinern wird es zum guten Teil nicht gern gesehen, dass dem in seiner Praxis sonst so souveränen Arzt in dieser Weise über die Schulter geblickt wird. Es sind aber nicht nur viele Mediziner, denen die kritischen Blicke der EBM-Evaluatoren zu einem Ärgernis geraten, sondern auch viele Naturheilkundler und Komplementärmediziner lassen es sich nicht gern gefallen, mit ihrem Handeln vor ein wissenschaftliches Beweisgericht gezogen $\mathrm{zu}$ werden. Manche fühlen sich sogar benachteiligt, weil sie die Wissenschaftlichkeit für einen Wesenszug der herrschenden Medizin halten. Tatsächlich sind z.B. Wirksamkeitsnachweise in der Naturheilkunde teilweise noch erheblich schwerer zu führen als dort. Die «Forschende Komplementärmedizin und klassische Naturheilkunde» hat sich allerdings von Anfang an nicht darauf einlassen wollen, hier irgendwelche Ausnahmen zu beanspruchen und die Wissenschaftlichkeit den Medizinern zu überlassen.

Grundsätzlich gibt es die Möglichkeit, die Wissenschaftlichkeit entweder sowohl für die herrschende Medizin als auch für die Komplementärmedizin und klassische Naturheilkunde aufrechterhalten zu wollen oder diese Forderung für beide fallen zu lassen. Die zweite Möglichkeit würde der ärztlichen Urteilskraft wieder den Raum geben, den sie wohl eigentlich verdient, und ist keineswegs von vornherein abzuweisen. Allerdings wären die meisten Ärzte damit wohl überfordert. Ich sehe aber noch einen andern Grund, warum wir uns auch in der Komplementärmedizin und klassischen Naturheilkunde der wissenschaftlichen Kontrolle nicht zu entziehen versuchen sollten. Dieser Grund ist, dass die herrschende Medizin sich in gewissen Grenzen - zwar auf die Heilung von akuten Krankheiten versteht, nicht aber darauf, wie man gar nicht erst krank wird, also auf das gesunde Leben. Auch die Öffentlichkeit erwartet von dem Gesundheitswesen lediglich diejenigen Vorkehrungen, die getroffen werden können, damit wir im Krankheitsfall möglichst leicht und schnell wieder gesund bzw. «gesund gemacht» werden. Dementsprechend pflegt das «Gesundheitsministerium» die politischen Voraussetzungen für die Heilung von Krankheiten zu schaffen, obwohl die Tätigkeit anderer Ministerien für die Gesundheit der Bevölkerung tatsächlich von viel grösserer Bedeutung ist. Beispielsweise ist die Häufigkeit von Herzinfarkten und andern Krankheiten bei Beschäftigten, deren Tätigkeit nur wenig Anerkennung findet und auch hinsichtlich der Gestaltungsspielräume unbefriedigend ist, drei- bis viermal so hoch wie bei denen, die mit ihrer Arbeit zufrieden sind und sie als einen Teil ihres

\begin{tabular}{ll}
\hline KARGER & (c) 2005 S. Karger GmbH, Freiburg \\
$\begin{array}{ll}\text { Fax +49 7614520714 } & \text { Accessible online at: } \\
\begin{array}{l}\text { E-mail Information@Karger.de } \\
\text { www.karger.com }\end{array} & \text { www.karger.com/fkm }\end{array}$
\end{tabular}

\section{KARGER}

www.karger.com
Prof. em. Dr. Klaus Michael Meyer-Abich 
eigenen Lebens erfahren, d.h. sich mit ihrer Arbeit identifizieren. Ist die Qualität der Arbeitsplätze unter diesen Umständen nicht viel wichtiger als die ganze Medizin?!

Niemand wird den Vorkehrungen zur Behandlung von Krankheiten ihre Bedeutung und ihre Berechtigung absprechen. Sie als «das» Gesundheitswesen zu verstehen verrät jedoch eine grobe Unterschätzung all der anderen Faktoren, von denen unsere Gesundheit abhängt. Dementsprechend machen sich die heutigen Mediziner zwar Gedanken über fettarme Nahrungsmittel oder Cholesterinsenker, lassen darüber aber munter ausser Acht, dass viel mehr Menschen durch den Stress am Arbeitsplatz oder durch Vereinsamung im Alter zu Tode kommen als durch Cholesterin [2].

Wohin gehören nun aber die Komplementärmedizin und die klassische Naturheilkunde? Dienen sie der Gesundheit in einem umfassenden Sinn oder ergänzen sie nur das heutige Krankheitswesen, das sich fälschlicherweise als ein Gesundheitswesen ausgibt? Auch uns soll niemand die Erfolge bei der Heilung von Krankheiten bestreiten - besonders derer, mit denen die herrschende Medizin nicht oder nicht so gut zurechtkommt. Wollen wir die klassische Medizin aber nur in ihrer Krankheitsorientierung komplementieren?

Dass Ärzte sich im Wesentlichen mit Krankheiten beschäftigen, hat sich erst mit der Verwissenschaftlichung der Medizin - bzw. mit diesem Anspruch - ergeben, denn (begrenzte) Störungen sind viel leichter zu verstehen als das Leben selbst. Jahrtausende zuvor hatte es die Medizin - von Hippokrates und Galen bis Boerhave - nicht nur mit Krankheiten zu tun, sondern mit dem gesunden Leben. Wo Krankheiten behandelt wurden, geschah dies grundsätzlich auf eine ganzheitliche Weise, also vom gesunden Leben im Ganzen von Natur und Gesellschaft her. In dieser Tradition stehen sowohl der legendäre Hausarzt als auch die klassische Naturheilkunde, und die Komplementärmedizin sollte sich unseres Erachtens ebenfalls nicht so verstehen, dass sie die herrschende Medizin nur in ihrer einseitigen Krankheitsorientierung ergänzt. Das hiesse: Komplementärmedizin und klassische Naturheilkunde sind Teil eines umfassenden Gesundheitswesens, nicht nur des herrschenden Krankheitswesens. Sie haben es vor allem mit dem gesunden Leben bzw. der Aufrechterhaltung der Gesundheit und nur mittelbar mit Krankheiten zu tun. Komplementärmedizin komplementiert die herrschende Medizin auch und vor allem, indem sie deren Krankheitsorientierung um die fehlende Gesundheitsorientierung ergänzt.
Die hier vorgeschlagene Erweiterung des Verständnisses von Komplementärmedizin wäre ein Befreiungsschlag gegenüber der Verengung durch die Krankheitsorientierung der herrschenden Medizin, die uns durch die Anforderungen der EBM zusätzlich in die Enge treibt. Zugleich wird für die Komplementärmedizin ein wesentlich grösserer Anspruch erhoben, als der der bloss sozusagen «hilfsdienstlichen» Ergänzung des Üblichen. Allerdings geht dieser Anspruch nicht so weit, nun auch noch entscheiden zu wollen, welche Krankheiten eigentlich nur Symptome für ungesunde gesellschaftliche und Lebensverhältnisse sind, sondern dies bleibt eine gemeinsame politische Begrenzung von Krankheits- und Gesundheitsmedizin. Die Erweiterung unseres Selbstverständnisses hätte weit reichende Konsequenzen für die Ansprüche der EBM an Naturheilkunde und Komplementärmedizin. Es läge uns fern, diese Ansprüche zurückzuweisen, aber wir dürften zu Recht erwarten, an unseren eigenen Zielen gemessen zu werden und nicht an denen der Krankheitsmedizin. Wenn unser Ziel vor allem die Aufrechterhaltung der Gesundheit ist, müssten unsere Methoden sich gerade hierbei bewähren. Die EBMgemässe Evaluation hätte sich ihrerseits also selbst zunächst einmal daran zu bewähren, dass Kontrollmöglichkeiten für den Zielerfüllungsgrad «Aufrechterhaltung der Gesundheit» entwickelt werden. Wir können und wollen dieser methodischen Entwicklung nicht vorgreifen, könnten uns aber vorstellen, dass z.B. die Intaktheit des Immunsystems sich als einer von mehreren passenden Indikatoren erwiese. Ausserdem werden Komplementärmediziner und Vertreter der klassischen Naturheilkunde natürlich gern bereit sein, mit ihrer Erfahrung zur Entwicklung geeigneter Indikatoren beizutragen, denn an Wissenschaftlichkeit wollen ja auch wir es nicht fehlen lassen.

\section{Literatur}

1 Evans RG, Stoddart GL: Producing health, consuming health care; in Evans RG, Barer ML, Marmor TR (eds): Why Are Some People Healthy and Others Not? The Determinants of Health of Populations. New York, Aldine de Gruyter, 1994, pp 27-64.

2 Lomas J, Contandriopoulos A-P: Regulating limits to medicine: towards harmony in public- and self-regulation; in Evans RG, Barer ML, Marmor TR (eds): Why Are Some People Healthy and Others Not? The Determinants of Health of Populations. New York, Aldine de Gruyter, 1994, pp 253-283.

3 Meyer-Abich KM: Vom Nutzen der Medizin - Wofür sind Ärzte, Medikamente, Krankenkassen, Krankheiten und Genesungen gut? Scheidewege 2002/2003;32: $377-405$. 